curred in a second, without explanation in spite of extensive investigation. Neither a malignant nor an infectious process was identified by multiple biopsies and cultures. Although the lesions were not typical of genital herpes and the cultures and results of histologic examination for herpes simplex virus were negative, empiric trials of acyclovir were given; this therapy was ineffective in both patients. Common causes of discrete vaginal ulceration such as trauma, foreign body, or malignancy were not present. Other infrequent causes of ulceration of the vagina include erosive lichen planus, pemphigus, pemphigoid, and Behçet's disease. None of these diagnoses was suggested by biopsy. Behçet's disease cannot be excluded but is extremely unlikely given the absence of recurrent ulceration and lack of vulvar or oral lesions. No other systemic manifestations of Behçet's disease were present, and the chronicity of a single ulcer in the vagina in patient 2 makes this diagnosis unlikely. Patient 1 had no evidence of inflammatory bowel disease as an explanation for the rectovaginal fistula.

The two patients described here most closely resemble patients with advanced HIV infection with idiopathic esophageal ulceration. Esophageal ulcers are chronic and painful, often invade adjacent structures, and may respond to systemic corticosteroid therapy. Both of our patients received systemic corticosteroids and demonstrated marked improvement, with complete healing of the lesion in the second patient. Several years ago Covino and McCormack $^{1}$ reported a single case of idiopathic vaginal ulceration in a woman with HIV infection, which responded to zidovudine; with this exception we are aware of no other reported cases of spontaneous vaginal ulcers or rectovaginal fistulas in women with HIV infection.

In summary, one HrV-infected woman with an extremely low $\mathrm{CD}^{+}$lymphocyte count was seen with an idiopathic vaginal ulceration and another had development of a rectovaginal fistula. Extensive culture and histologic studies of the involved vaginal tissue failed to identify an infectious or microbial cause, although direct involvement by HIV cannot be excluded. Similarly an etiologic role for antiretroviral drugs, although unlikely, is possible. High-dosage systemic corticosteroid therapy appears to have contributed to the healing of the vaginal ulcer in one patient and stabilization of the fistula in the other; however, the optimal dose and duration of therapy remain undetermined. Clinicians should be on the alert for unusual lower genital tract ulcerative lesions in women with advanced HIV disease.

\section{REFERENCES}

1. Covino JM, McCormack WM. Vulvar ulcer of unknown etiology in a human immunodeficiency virus-infected woman: response to treatment with zidovudine. AM J OBSTET GYNECOL 1990;163:116-8.

2. Indorf AS, Pegram PS. Esophageal ulceration related to zalcitabine (ddC). Ann Intern Med 1992;117:133-4.

\title{
Clinical significance of placenta-confined nonmosaic trisomy 16
}

\author{
Helen Brandenburg, MD, PhD, Frans J. Los, MD, PhD, and Peter In't Veld, PhD \\ Rotterdam, The Netherlands
}

Since the introduction of chorionic villi sampling in 1984 we have observed five first-trimester cases of nonmosaic placenta confined trisomy 16. At subsequent follow-up it appeared that all five had an adverse maternal or fetal outcome. In three cases the mother had preeclampsia. Two of the infants were born with multiple congenital abnormalities, including tetralogy of Fallot. All children were growth retarded. (Aм J OBSTET GYNECOL 1996;174:1663-4.)

Key words: Prenatal diagnosis, trisomy 16, confined placenta abnormality, chorionic villus sampling

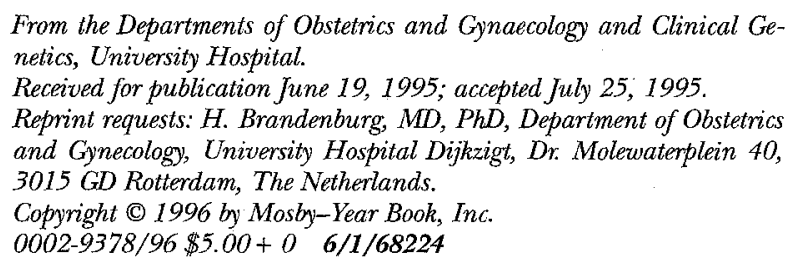

From the Departments of Obstetrics and Gynaecology and Clinical GeReceived for publication June 19, 1995; accepted July 25, 1995. Reprint requests: $H$. Brandenburg, $M D, P h D$, Department of Obstetrics 3015 GD Rotterdam. The Netherlands. $0002-9378 / 96 \$ 5.00+0 \quad 6 / 1 / 68224$

One of the problems of cytogenetics in chorionic villi is the presence of chromosomal mosaicism or rare nonmosaic trisomies not present in amniotic fluid cells. An increased incidence of adverse neonatal outcome is reported by some authors, ${ }^{1}$ whereas others have not found any adverse effect in these cases. ${ }^{2}$ 


\section{Case reports}

Case 1. A 39-year-old woman, gravida 3, para 2, underwent chorionic villus sampling (CVS) at 10.4 weeks. A $47, \mathrm{XX}+16$ karyotype was found in all cells. Amniocentesis showed a normal 46,XX karyotype. At 37 weeks a 2300 gm female infant was born ( 10 th percentile), whereas the previous children had birth weights on the 50 th percentile. The infant showed no malformations.

Case 2. A 36-year-old primigravid woman underwent transabdominal CVS at 14.3 weeks. All cells revealed a $47, \mathrm{XY}+16$ karyotype, but the trisomy was not confirmed in subsequent amniocenteses. At 29.3 weeks she was admitted with pregnancy-induced hypertension, hemolysis, elevated liver enzymes, and low platelets (HELIP syndrome). Because her condition had deteriorated, a cesarean section was done at 31.6 weeks. A $1100 \mathrm{gm}$ male infant was born ( $3 r d$ percentile). The mother had severe but transient coagulopathy and could be discharged on the fifteenth postoperative day.

Case 3. The patient, gravida 6 , para 0 , underwent transabdominal CVS because of a $25 \%$ risk of (nonfragile $\mathrm{X}) \mathrm{X}$-linked mental retardation in male offspring. Previously she had two spontaneous abortions and three terminations because of a male fetus. The parental karyotypes were normal. Chorionic villi showed a 47,XX +16 karyotype, which was not found in amniocytes. From the sixteenth week onward severe growth retardation was noted. At 24 weeks a complex heart anomaly consistent with tetralogy of Fallot was seen. At 29 weeks the mother had preeclampsia and HELLP syndrome. At 33 weeks fetal death was established and shortly thereafter a 860 gm female infant was born ( $<3$ rd percentile). The girl had facial dysmorphism, tetralogy of Fallot with an atrial septum defect, abnormal pulmonary lobes, and simian creases. Unfortunately, karyotyping from various fetal tissues failed because of maceration of the fetus.

Case 4. A 38-year-old woman, gravida 6, para 3, aborta 2, underwent transabdominal CVS at 11.3 weeks. Analysis of the chorionic tissue revealed trisomy 16 , which could not be confirmed with amniocentesis. At 40 weeks a 2500 gm female infant was born (10th percentile). She was diagnosed with tetralogy of Fallot, scoliosis with three fused vertebrae, and fused ribs. She has normal mental development but receives treatment for speech problems.

Case 5. At 12 weeks this 40 -year-old woman, gravida 5 , para 2, aborta 2, underwent transabdominal CVS and had a confirmatory amniocentesis at 16 weeks. A placentaconfined 47,XX +16 karyotype was found. At 34 weeks hypertension and deteriorating renal function were diagnosed. At 37.5 weeks labor was induced and a $2880 \mathrm{gm}$ female infant was born (25th percentile). The mother recovered rapidly after the delivery.

\section{Comment}

Because placental dysfunction can lead both to intrauterine growth restriction and preeclampsia, it is conceivable that in the case of an abnormal placental karyotype the normal process of placentation and trophoblast invasion of the spiral arteries is disturbed. Currently there is sufficient evidence that this mechanism plays a role in at least trisomy $16 .^{\jmath, 3}$ Less is known about the clinical relevance of other placenta-confined rare trisomies. Because most placenta-confined trisomy 16 represents the corrected state of a trisomic conception with an extra maternal chromosome 16 , with a high chance of maternal uniparental disomy, ${ }^{1,3}$ it cannot be excluded that a low mosaic trisomy in the fetus (not found in amniocentesis) or the uniparental disomy per se could have caused the congenital abnormalities in two of our newborns.

In our opinion a pregnancy in which a placenta-confined nonmosaic trisomy 16 is established should be considered at high risk for maternal or fetal complications, or both.

\section{REFERENCES}

1. Kalousek DK, Langlois S, Barret I, Yam I, Wilson DR, HowardPeebles PN, et al. Uniparental disomy for chromosome 16 in humans. Am J Hum Genet 1993;52:8-16.

2. Dorfmann AD, Perszyk J, Robinson P, Black SH, Schulman JD. Rare non-mosaic trisomies in chorionic villus tissue not confirmed at amniocentesis. Prenat Diagn 1992;12:899-902.

3. Wolstenholme J. An audit of trisomy 16 in man. Prenat Diagn 1995; 15:109-21. 